\title{
Directives pédagogiques et soubassement idéologique
}

La littérature à l'école sénégalaise, de l'époque coloniale à aujourd'hui

Educational directives and ideological basis. Literature at Senegalese schools, from the colonial era to the present day

Directivas pedagógicas y fundamentos ideológicos. La literatura en la escuela senegalesa, de la época colonial a la actualidad

\section{Abdoulaye Elimane Kane}

\section{(2penEdition}

\section{Journals}

Édition électronique

URL : https://journals.openedition.org/ries/2685

DOI : 10.4000/ries.2685

ISSN : 2261-4265

\section{Éditeur}

France Education international

Édition imprimée

Date de publication : 1 décembre 2012

Pagination : $91-100$

ISSN : 1254-4590

Référence électronique

Abdoulaye Elimane Kane, " Directives pédagogiques et soubassement idéologique », Revue internationale d'éducation de Sèvres [En ligne], 61 | décembre 2012, mis en ligne le 06 février 2015, consulté le 06 juillet 2021. URL : http://journals.openedition.org/ries/2685 ; DOI : https://doi.org/ $10.4000 /$ ries.2685 


\section{Directives pédagogiques et soubassement idéologique}

\section{La littérature à l'école sénégalaise, de l'époque coloniale à aujourd'hui}

\section{Abdoulaye Elimane Kane}

L'introduction de la littérature à l'école, au Sénégal, est repérable à travers deux séquences historiques: la fonction assignée aux programmes des écoles publiques par l'administration coloniale; la promotion de la littérature africaine d'expression française comme volonté de rupture, de réhabilitation et d'émancipation des pays africains indépendants. Dans les deux situations, les démarches sont soutenues par un projet qui vise à forger, en même temps que des outils d'accès au savoir par le moyen du français - et non des langues nationales - une conscience apte à servir un dessein : la réalisation d'un type d'homme. Pour illustrer ces deux séquences historiques seront analysées dans cet article d'abord deux séries de manuels emblématiques concernant l'école primaire - avec une longévité remarquable pour ceux conçus sous l'administration coloniale et maintenus officiellement un peu plus d'une décennie après les «indépendances »-, témoins d'une façon d'enseigner et de former les esprits. Seront ensuite examinés, à propos du collège et du lycée, les effets de réformes successives de l'enseignement de la littérature liées aux exigences d'une époque et aux attentes d'une génération nouvelle.

L'approche privilégiée dans cette présentation porte sur les intentions qui gouvernent le choix des auteurs et des extraits d'œuvres littéraires, en somme, sur les préoccupations d'ordre politique et/ou idéologique des institutions et des auteurs de manuels. Elle peut paraître arbitraire dans la mesure où décideurs et autorités scolaires des deux périodes insistent sur la maîtrise de la langue comme objectif principal de l'étude de la littérature. Toutefois, cette option trouve sa justification aussi bien dans les orientations officielles que dans les arguments qu'exhibent en ouverture de leurs manuels respectifs auteurs français et africains. Tous s'accordent sur deux points : conduire l'élève vers une maîtrise progressive du français et choisir en priorité des textes littéraires se rapportant à la réalité et à des valeurs africaines. Ces orientations exhortent le maître à s'ouvrir aux œuvres relevant des genres les plus variés de la littérature, appartenant à différentes aires culturelles, une fois qu'elles sont traduites en français. Quand bien même le diptyque enracinement/ ouverture finit par connaître un certain essor, au nom de la quête de valeurs universelles, les textes destinés à illustrer l'africanité ou la question identitaire africaine demeurent un lieu d'investissement idéologique considérable. 


\section{LITTÉRATURE COLONIALE ET REGARD ETHNOGRAPHIQUE : LA SÉRIE DES MAMADOU ET BINETA}

L'expression « littérature coloniale » a été forgée et revendiquée par des écrivains français ayant vécu et exercé des responsabilités diverses en Afrique, au cours de la période coloniale. Par la création de l'" Association des écrivains d'expression française de la mer et de l'outre-mer » et du «Grand prix de la littérature coloniale » ils marquaient leur territoire. C'est aux œuvres de ces auteurs - dont il fait lui-même partie - que, dans un premier temps, André Davesne, auteur de la série de manuels Mamadou et Bineta, emprunte les textes destinés aux exercices de lecture figurant dans ses manuels.

\section{Les historiettes du syllabaire Mamadou et Bineta}

Conçu et réalisé à l'intention des élèves africains de l'école primaire par A. Davesne, premier inspecteur et chef du service de l'enseignement primaire au Sénégal dans les années 1930, le syllabaire Mamadou et Bineta permet d'atteindre des résultats rapides en matière de lecture. Ce manuel allie une iconographie abondante à des exercices de lecture de courts paragraphes - des " historiettes »- aux différents stades du processus d'acquisition du vocabulaire. Ces petits textes utilisent divers procédés de mise en scène : dialogues, descriptions de personnages, d'actions et de situations. On notera que Davesne est lui-même un écrivain, auteur d'un ouvrage sur la vie africaine, intitulé Croquis de Brousse. Ces historiettes peuvent donc être regardées comme des éléments ou une épure d'une pratique littéraire effective, dont des illustrations figurent dans Mamadou et Bineta sont devenus grands.

À l'objectif didactique de ces historiettes s'ajoute un autre objectif, fondé sur une démarche ethnographique : scènes de la vie quotidienne africaine, objets, noms de personnes (Moriba, Toto), situations renvoyant à une psychologie réputée africaine ou encore à ce qu'on appelle «l'âme africaine ». Enfin, ces historiettes composées d'un thème, d'une intrigue et d'une conclusion recèlent explicitement ou implicitement un enseignement: hygiène corporelle, critique de la paresse, valeur de l'école, métiers, joies et peines, etc. Pour certains analystes, l'intention et le résultat visés à travers la morale de ces historiettes sont strictement de l'ordre de la formation civique. Pour d'autres, le projet pédagogique et sa mise en œuvre, notamment le contenu de ces historiettes, traduisent la culture "scientifique » dominante de l'époque : l'ethnographie. Cette discipline marque ses objets par un culte de la différence. Aussi, dans les "sociétés indigènes » qu'elle étudie, cherchet-elle ce qui en fait l'essence et, dans le cas de l'Afrique, «l'âme africaine». Son influence sur le décideur politique et concepteur de manuel destiné aux écoliers africains est avérée. Ces lectures divergentes mettent toutes l'accent sur le contenu 
des textes davantage que sur les aspects formels de ceux-ci. Certaines de ces historiettes, gardées par la mémoire collective, fonctionnent comme des images d'Épinal. Ainsi, le textes et les images de "La case de Birima brûle " sont devenus une métaphore usuelle et un code d'appel à la solidarité. Autre image : la construction du personnage de Toto, prototype du petit garçon africain de brousse, sympathique, souvent puni pour ses écarts, et par extension et construction tardive, une sorte d'hurluberlu, amusant et incertain.

\section{La méthode d'opposition}

Mamadou et Bineta sont devenus grands (cours moyen), propose des extraits d'œuvres littéraires (prose et poésie). La présentation des textes en fonction de leur contenu relatif aux us, coutumes et systèmes de représentation des Africains confirme l'omniprésence du regard ethnographique.

Ces textes sont dus, dans leur écrasante majorité, à des auteurs coloniaux qui déclarent exprimer un réalisme colonial à travers des récits objectifs tirés d'une expérience directe, comme l'indiquent des titres d'ouvrages tels que : Croquis de Brousse; Visions d'Afrique; Les Paysans noirs; Noirs et Blancs; Le livre de la brousse; À l'ombre de mon baobab; Magie noire. On y trouve des extraits d'œuvres d'auteurs français connus : Alphonse Daudet, André Demaison, Pierre Loti, Jérôme et Jean Tharaud, André Theuriet.

À ce premier trait s'ajoute, comme pour le confirmer, le recours à la méthode d'opposition : présenter successivement un "modèle » de vie à l'africaine et un "modèle" de vie à la française. Le traitement du thème du village constitue un exemple tout à fait représentatif du procédé. Deux textes en donnent une bonne illustration : Un village de brousse (A. Davesne, notre auteur de manuel) et Un pauvre village de montagne (Ramuz). Les descriptions révèlent l'extrême dénuement de ces deux villages et, en même temps, leurs différences profondes. La méthode d'opposition s'applique à des sujets si divers qu'elle ne peut manquer de vouloir signifier quelque chose de structurel : la géographie et les traits ethniques, les caractères raciaux et des invariants culturels.

Ce manuel contient également des compositions françaises d'élèves, de bonne facture, ne portant cependant que sur des sujets locaux. Il se termine par un chapitre consacré à des extraits de contes, légendes et fables.

\section{NouveauX REgARdS SUR LA FORME ET LE CONTENU DES TEXTES}

L'introduction de la littérature dans l'enseignement au Sénégal n'a pas suivi un itinéraire rectiligne ni obéi à une périodisation opposant de manière radicale tous les aspects de la période coloniale à tous ceux de la période post coloniale. D’un côté, les rééditions successives des Mamadou et Bineta ont été l'occasion d'adaptations à de nouvelles données politiques, sociales et culturelles ; 
des romans comme Batouala de René Maran, Le roman d'un Spahi de Pierre Loti, Les Randonnées de Samba Diouf des frères Tharaud ne sont pas considérés par la critique comme appartenant au « colonialisme littéraire ». D'un autre côté, les œuvres des premiers romanciers africains restent sous l'influence des auteurs français, voire de l'idéologie coloniale, comme le prouve, par exemple, Force Bonté du Sénégalais Bakary Diallo. De même, les manuels conçus par des auteurs sénégalais n'ont pas immédiatement rompu avec les thématiques et le mode d'agencement des extraits d'œuvres littéraires propre aux livres de Davesne. Il est vrai que, progressivement, la volonté de rupture s'est affirmée, à la fois du point de vue du contenu et de celui de l'intérêt pour les caractéristiques formelles. C'est de ces constantes et de ces changements dont nous voulons maintenant rendre compte.

\section{Le début de la prise en compte de la valeur littéraire d'un texte}

À la faveur d'une ultime réédition du manuel Mamadou et Bineta lisent et écrivent couramment, Davesne introduit deux innovations qui témoignent d'un changement de critère de choix des textes littéraires. «Depuis la première édition [...], écrit-il dans son avant-propos, l’Afrique a évolué considérablement. Une ère nouvelle s'ouvre pour le pays. » L'auteur ajoute plus loin, à propos de la 94 nouvelle édition : «elle s'est débarrassé de tout ce qui, dans la perspective d'évolution du pays, n'aurait pas manqué d'apparaître comme périmé et dépassé. »

Dans ses «Conseils au maître », Davesne insiste sur le critère de « qualité littéraire » qui a guidé le choix des textes consacrés à la récitation. Il parle de «bons auteurs ", dont les poèmes doivent être "lus et récités de manière expressive »; l'un des objectifs de cet exercice étant de faire en sorte que l'élève soit " charmé par le rythme et l'harmonie du poème ». On trouve dans ce manuel de $1^{\text {re }}$ et $2^{\mathrm{e}}$ années du cours élémentaire un grand nombre d'extraits de poèmes de Victor Hugo : La ronde, Lazzara, L'enfant rêve, Jeu d'enfant. Mais aussi ceux d'autres auteurs français : Maupassant (La Chanson du rayon de lune); La Fontaine (Le Héron) ; Sully Prudhomme (La Pluie); Alfred de Musset (Le Sommeil de l'enfant), etc.

La deuxième innovation réside dans le fait que chacun des onze chapitres s'achève par la présentation d'un conte ou d'un récit, la plupart du temps africain, qui doit être lu mais n'a pas d'autre but que celui de distraire l'élève.

\section{Nouveaux départs, nouvelles littératures et nouvelles didactiques}

En dépit de la longévité de la série des Mamadou et Bineta (créés en 1931 et maintenus jusque dans les années 1970), ces quarante dernières années ont vu naître et se multiplier au Sénégal de nouveaux manuels, dont les thèmes dominants sont d'abord l'enracinement/réenracinement, puis l'ouverture, et enfin les 
enjeux des mutations de notre époque. Quelques titres de nouveaux manuels de lecture à l'école primaire en attestent: Afrique mon Afrique; Je lis et j'écris ; Sidi et Rama ; Ami et Rémi ; Soleil d'Afrique; Mon unique livre de français ; Les Manuels de français (Ineade); Les Cahiers d'activité en français; Album de lecture.

\section{L'ombre portée de Senghor et de la théorie de la Négritude}

Senghor définit la Négritude comme l'« ensemble des valeurs de civilisations du monde noir. " Il s'est efforcé toute sa vie de traduire cette vision dans ses écrits et dans son action politique comme Président de la République sénégalaise. Motivés par une volonté de contester les préjugés sur l'Afrique et les Africains et de réhabiliter l'homme noir et sa civilisation, le mouvement et la théorie de la Négritude ont ouvert la voie à des productions littéraires prônant la lutte anticoloniale et l'enracinement dans les cultures nègres. L'Anthologie de la nouvelle poésie africaine et malgache de langue française de Senghor (préfacée par «Orphée noir» de Sartre) est considérée comme l'un des textes fondateurs de la nouvelle littérature nègre d'expression française et comme le point de départ de la reconnaissance de cette littérature.

La maison d'édition d'Ami et Rémi se réjouit, dans sa brève présentation, de constater que les quatre auteurs du manuel ont réalisé cet ouvrage "dans le respect de la " "Table des valeurs" africaines », avant d'ajouter : " nos deux enfants de Colibatan, ouverts aux souffles fécondants venus de l'extérieur, mais tout d'abord profondément enracinés dans leur terroir, ne seront pas des extravertis ». «Enracinement » et « ouverture », deux maîtres-mots de la pensée senghorienne.

\section{Des textes-phares pour exprimer l'être-au-monde de l'Africain}

\section{Les textes en prose dans Ami et Rémi}

Le principe d'enracinement et d'ouverture s'y traduit sous la forme d'un recours à des extraits d'œuvres d'auteurs français et africains, sélectionnés en fonction du thème retenu, et suivis d'exercices de vocabulaire, de grammaire, de dictée et de composition française. Les auteurs africains y sont plus présents que les auteurs non africains. Parmi eux, des figures féminines apparaissent, comme Mariama Ba dont le roman Une si longue lettre est devenu un classique de la littérature africaine ou Nafissatou Diallo (Awa, la petite marchande), et bientôt Aminata Sow Fall (L'appel des arènes). D'autres œuvres comme celle de Cheikh Hamidou Kane (L'Aventure ambiguë), Sembène Ousmane (Le mandat), Senghor, Birago Diop, Aimé Césaire, pour n'en citer que quelques unes, figurent désormais au programme d'enseignement de la littérature, à l'école primaire, au secondaire et à l'université. Des extraits d'œuvres d'auteurs de la diaspora noire sont également proposés dans Ami et Rémi: Léon G. Damas (Veillées noires), René Maran (Le livre de la brousse), Joseph Zobel (La rue Cases-Nègres). Enfin, 
des textes d'écrivains européens figurent aussi en bonne place dans ce manuel : Marcel Pagnol (La Gloire de mon père), Robert Delavignette (Les Paysans noirs), Léon Tolstoï (Les Quatre livres de lecture).

\section{Les récitations dans Ami et Rémi}

Les auteurs ont regroupé en fin de volume des poèmes, sous la rubrique « récitation ». Ce choix spatial porte la marque d'une intention : mettre en relief des textes destinés à un type d'exercice bien déterminé. Ce sont des poèmes écrits par des auteurs africains et noirs de la diaspora, de grande renommée. Leur contenu globalement porte sur l'Afrique ou une de ses figures emblématiques et utilise pour l'évoquer un discours s'appuyant sur différentes formes de la célébration. La louange: "Afrique, mon Afrique, / Afrique des fiers guerriers dans les savanes ancestrales»(David Diop). La nostalgie: «Joal, / je me rappelle, / Je me rappelle les fastes du Couchant / Où Koumba Ndofène voulait faire tailler son manteau royal. »(L. S. Senghor). Les ressources positives de l'autodérision: par exemple ce poème de l'Ivoirien Bernard Dadié : "Je vous remercie mon Dieu de m'avoir créé Noir / d'avoir fait de moi la somme de toutes les douleurs, / mis sur ma tête le monde ». Ou bien encore ce poème : Prière du petit enfant nègre de l'antillais Guy Tirolien, qui, après s'être exclamé : "Seigneur je ne veux plus aller à leur école", oppose les vertus du village ancestral aux désagréments de la ville: «Triste comme ce messieurs de la ville / Ces messieurs comme il faut / Qui ne savent plus danser le soir au clair de lune / Qui ne savent plus marcher sur la chair de leurs pieds / Qui ne savent plus conter les contes aux veillées. » Une preuve de plus de l'importance du thème du déracinement appelant du même coup le réenracinement.

Ces poèmes ont beaucoup de succès à l'école et dans l'opinion cultivée, comme par exemple ce poème de David Diop, cité plus haut, que l'on aime déclamer avec d'autant plus de passion et d'application que le poète a disparu tragiquement dans un accident d'avion, au large de Dakar, en « rentrant au pays ». Il en est de même des deux poèmes de Senghor les plus lus, connus, et cités: Joal et Femme noire. Ces poèmes sont de véritables hymnes à l'Afriquemère. Ils sont l'expression d'une nouvelle manière d'écrire vouée à faire prendre conscience des défis d'une Afrique nouvelle.

\section{L'ère des réformes de l'enseignement de la littérature}

\section{L'exemple de Sidi et Rama}

Le manuel Sidi et Rama, réalisé par l'Institut national d'étude et d'action pour le développement de l'éducation (Ineade), porte la marque d'une volonté politique nouvelle. Dans leur avant-propos, les auteurs annoncent des «textes et poèmes d'auteurs confirmés ». Ils sont d'avis que «la lecture doit être un moment de découverte et un moment de plaisir », et insistent sur le caractère pratique et 
utilitaire du manuel. Ces directives témoignent d'une volonté d'appliquer les recommandations des États généraux de l'éducation et de la formation (1981), à l'avènement du Président Abdou Diouf, et dont les conclusions forment la réponse à la crise de l'École sénégalaise considérée alors comme peu tournée vers les réalités africaines et les questions de développement durable. Les thèmes qui structurent Sidi et Rama affichent une volonté d'innovation et d'ancrage dans des réalités contemporaines porteuses de signes de mutations politiques, sociales et culturelles. Le texte de Sembène Ousmane intitulé «La marche des femmes », et extrait de son roman Les bouts de bois de Dieu, s'inspirant de la longue grève des cheminots de la ligne de chemin de fer Dakar/Bamako, en constitue une figure emblématique.

Les extraits d'œuvres de romanciers, de poètes et de conteurs s'efforcent de donner une illustration vivante de ce nouveau contexte. Ainsi, ce texte qui dépeint de manière saisissante la découverte brutale de la ville de Dakar par un jeune paysan est suivi d'un exercice d'expression écrite sur la différence entre ville et village et projette la thématique de l'acculturation dans son double sens de rencontre violente et d'enrichissement mutuel entre deux cultures.

On trouve aussi dans ce manuel un large spectre d'œuvres appartenant à la littérature francophone. Outre ceux de Bernard Dadié de Côte d'Ivoire, de Seydou Badian du Mali, de Djibril Tamsir Niane de Guinée, de L.S. Senghor et Birago Diop du Sénégal, sont proposés des textes d'auteurs appartenant à différentes aires culturelles du monde francophone comme Michelle Lagabrielle (Les colombes du Liban), Michel Pellaton et J.-P. Jaubert (Histoire de la presse écrite), Laura Makarius, Raoul Makarius (Anthologie de la littérature arabe contemporaine, Le Roman et la Nouvelle), Simone Weil (La condition ouvrière), Michel Tournier (Vendredi ou la vie sauvage).

Sidi et Rama présente, en fin de volume, dix-neuf proverbes et treize devinettes, un conte de Birago Diop extrait des Contes d'Ahmadou Koumba ou bien encore cette légende "La parole du griot » de Mamadou Kouyaté, transcrit par D. T. Niane dans Soundjata ou l'épopée mandingue.

L'analyse des textes d'Ami et Rémi d'une part, et de Sidi et Rama d'autre part confirme l'importance des deux critères qui fondent le choix des extraits d'œuvres littéraires : "contenu » et «qualité littéraire ». Le premier ressortit à ce que nous désignons comme le soubassement idéologique de la conduite des pédagogues et des décideurs, le second au seul souci de valeur expressive de la langue des auteurs comme mode final de distinction entre ce qui relève strictement de la littérature et ce qui s'en distingue.

\section{Réformes successives dans le cycle secondaire}

L'enseignement de la littérature au collège et au lycée, dont l'objectif est, à partir de la classe de $6^{\mathrm{e}}$, de consolider les acquis de l'école élémentaire et d'élever progressivement le niveau de maîtrise du français, a fait l'objet de plusieurs réformes pour un motif récurrent : l'adaptation au contexte sénégalais. 
Les étapes successives qui vont de la réforme de 1972, décidée par la Conférence des ministres de l'Éducation nationale des États d'expression française à celle de 1995-1998, faisant suite au constat d'insuffisance des Recommandations des états généraux de l'éducation et de la formation (1981), prouvent que c'est le degré de prise en compte de chacun de ces deux critères qui fait problème. En effet, les auteurs de manuels de lecture appliquant les directives de 1972 présentent la "qualité littéraire » comme étant «le premier critère du choix des textes ». La distribution des thèmes et l'intitulé des sous-thèmes renvoient de toute évidence au contenu comme autre critère déterminant : "Les racines de l'homme négro africain » (classe de $3^{\mathrm{e}}$ ); « Le déracinement » (classe de seconde); "L'engagement» et «l'Afrique des Indépendances » (classes de première et terminale). S'agissant par exemple du déracinement - occasion d'analyser tous les aspects de l'acculturation -, quatre sous-thèmes confirment l'intérêt pour le contenu : "Les tentations de la ville»; "Les chemins de l'Europe»; "Les conflits de culture "; "Les métamorphoses de l'individu », sous-thèmes pour l'étude desquels des textes de grands auteurs sont sélectionnés : Cheikh Hamidou Kane, Ferdinand Oyono, Chinua Achébé, Mongo Béti, Aimé Césaire, Birago Diop, etc.

Un nouveau tournant s'est opéré à la faveur des nouvelles orientations préconisées par la Commission nationale du français à partir de 1998. Pour cette dernière, la Loi d'orientation et les Conclusions issues des États généraux de l'éducation et de la formation (1981), en dépit de leur volonté de remédier aux faiblesses du système éducatif sénégalais, n'ont pu réellement extraire l'enseignement du français de la situation de crise où il se trouvait. La réforme entreprise entend opérer « une rupture avec l'ancienne pédagogie et réconcilier l'enseignement du français avec la vie et la société sénégalaise ». Les rédacteurs rappellent que le français est, d'après des enquêtes sociologiques, langue seconde (L2) par rapport aux langues nationales, en dépit de son statut de langue officielle, de médium et de matière d'enseignement. D'où la nécessité d'une didactique appropriée à ce contexte de plurilinguisme : des éléments de la culture des élèves, le conte et la poésie en langues nationales par exemple, peuvent servir de point de départ pour l'enseignement de la littérature en français.

Le premier acte novateur de ce tournant se trouve dans la décision « de supprimer l'étude thématique qui est réductrice et limite les connaissances littéraires de l'élève » et de marquer une nette préférence pour «l'approche par les genres littéraires et la perspective historique en littérature africaine et française ». Sur ce point, l'innovation consiste à organiser la didactique autour de "grappes» de genres comprenant les éléments suivants, pour chaque niveau, de la $6^{\mathrm{e}}$ à la terminale : un genre littéraire prioritaire + quelques autres en complément + des extraits d'œuvres de littérature africaine, de littérature française et de littérature francophone. 
Des genres littéraires prioritaires sont distribués en fonction des niveaux d'enseignement: le conte et la poésie en $6^{\mathrm{e}}$; le théâtre et l'épopée en $5^{\mathrm{e}}$; le roman et le théâtre en $4^{\mathrm{e}}$; culture générale en $3^{\mathrm{e}}$ : des textes en rapport avec l'environnement de l'élève (défis de la modernité, drogue, sida, science et technologie etc.) ; le roman et la nouvelle en seconde; la poésie et le conte en première ; le théâtre et la critique littéraire en terminale.

Par exemple, en classe de $6^{\mathrm{e}}$, la grappe s'organise ainsi : un genre dominant, le conte et la poésie et, comme autres genres, les récits à caractère autobiographique dont les héros ont l'âge des élèves ; une œuvre intégrale, Le Pagne noir de Bernard Dadié ou les Contes du chat perché de Marcel Aymé ; des extraits de L'enfant noir de Camara Laye, La gloire de mon père de Marcel Pagnol. Aux deuxième et troisième trimestres : les élèves étudient la poésie (Birago Diop, Senghor) ainsi que le théâtre, des proverbes et des maximes.

Deuxième innovation de la Commission du français : accroître les compétences de l'élève, à partir de la classe de seconde, en matière d'analyse des œuvres littéraires : structure et langue d'un texte, type d'écriture, évolution d'un genre littéraire. Ainsi, tout en fixant à l'étude du roman et de la poésie, l'objectif "d'enraciner les élèves dans leur milieu », la Commission n'en insiste pas moins sur l'analyse et la maîtrise des aspects formels.

L'introduction à l'étude de l'image, de la $6^{\mathrm{e}}$ à la $3^{\mathrm{e}}$, constitue un autre aspect important de cette nouvelle orientation. L'image doit cette attention au fait qu'elle est partie intégrante de la culture moderne et accompagne tous les textes introductifs des manuels sénégalais de lecture. Elle est traitée comme langage à maîtriser dans le contexte d'apprentissage de la langue.

Par ailleurs, dans cette nouvelle démarche, un accent particulier est mis sur l'étude, en première et en terminale, des œuvres des précurseurs de la poésie de la négritude : Damas, Césaire, Senghor, Tirolien, Rabémananjara.

Enfin, il convient de signaler, dans cette réforme, la place accordée à l'initiation à l'histoire et aux procédés de la création romanesque en littérature africaine, avec notamment une périodisation qui identifie des œuvres phares de 1920 à 1945, puis de 1945 à 1960 et de 1960 à nos jours.

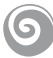

De ce parcours historique sur la littérature à l'école, au Sénégal, on peut retenir quelques repères. L'intérêt accordé à la dimension diachronique tient à deux faits essentiels. Le continent est passé de la littérature orale à la littérature écrite en cinquante ans - mais le souci de l'oralité (contes, récits, poésie) est demeuré omniprésent dans tous les manuels de lecture. Par ailleurs, le passage d'une littérature coloniale à une littérature de protestation et d'émancipation témoigne d'un rythme d'évolution lié notamment au champ des luttes politiques de chacune de ces périodes. 
La question des deux critères de choix des textes, fil directeur de cette contribution, permet de constater ceci : la référence à l'identité africaine à tous les stades, avec certes des regards différents, montre l'importance du contenu manifeste des textes, malgré les limites de celui-ci; l'accent mis, dans le cycle secondaire notamment, sur l'initiation des élèves aux différentes formes d'écriture et sur l'attention particulière accordée à l'esthétique de la poésie orale et des contes africains pour servir de point de départ à l'étude des genres dans les littératures africaine, française et francophone, est la preuve que le deuxième critère est tout aussi essentiel pour comprendre le rapport du particulier et de l’universel véhiculé par la littérature.

\section{Bibliographie \\ Quelques ouvrages de référence}

FOUET Francis et RENAUDEAU Régine (1988) : Littérature africaine. Le déracinement, NEAS, Dakar.

LY Boubacar (2009): L'École et les instituteurs, Tome 1, Les instituteurs au Sénégal de 1903 à 1945, L'Harmattan.

MOURALIS Bernard (1984): Littérature et développement. Essai sur le statut, la fonction et la représentation de la littérature négro-africaine d'expression française, Paris, édition Silex.

SAINVILLE Léonard. Anthologie de la littérature négro-africaine, romanciers et conteurs, Paris, Présence Africaine, tome 1, 1963, 456 p.; tome 2, 1968, 644 p.

JOUBERT Jean-Louis (dir.) (1992) : Littérature francophone - Anthologie, Paris, Nathan.

\section{Quelques œuvres d'auteurs sénégalais}

BA Mariama (1979) : Une si longue lettre, NEAS, Dakar.

DIOP Birago (1976): Contes d'Ahmadou Koumba, Présence Africaine.

KANE Cheikh Hamidou (1961) : L'Aventure ambiguë, Julliard, Paris.

SEMBÈNE Ousmane (1960): Les bouts de bois de Dieu, Plon.

SENGHOR L. S., Anthologie de la nouvelle poésie nègre et malgache de langue française, Présence Africaine, Paris, 1948. Préface de J.-P. Sartre, "Orphée Noir ». 